\title{
ON THE ROLE OF CHAOS AND INSTABILITY IN THE EVOLUTION OF NONLINEAR NONSTATIONARY STELLAR SYSTEMS
}

\author{
S.N. Nuritdinov \\ Physics Department, Tashkent State University, 700095, Tashkent, Russia
}

\begin{abstract}
The role of chaos and instability in evolution of nonlinear, non-stationary stellar system have been discussed. It is possible to distinguish between the only two different cases (1) strongly non-stationary stage when we have the violent relaxation accompanied by the compulsive mixing (ii) weakly non-stationary state or quasilinear cuse, when the quasidiffusion mixing takes place. In case (i), the chaos and chaotic motion $g$ stars play very important role and in case (ii) the role of any type of instability is important.
\end{abstract}

\section{ON STATES FAR-AWAY FROM THE STATIONARITY}

The construction of a theory of the nonstationary stage evolution of stellar systems requires, at least, the analysis of the following problems: (1) the study of relaxation mechanisms and the role of chaos phenomena, (2) physics of instability, occuring on the background of a nonlinear nonstationary system, (3) the classification of nonstationary states, nonlinear effects and the calculation of their characteristic times. The observed differences and large-scale structure of stellar systems can primarily be explained by the investigation of nonlinear nonstationary stage of their evolution. In this case the evolution character depends on the initial values of the nonstationarity degree, density distribution, degree of rotation as well as on the $t y p e s$ of instability and other factors. At the first the dynamical evolution of a nonstationary system is related with the process of relaxation and phase mixing [1-3]. But mechanisms of phase mixing and relaxation of nonlinear nonstationary stellar 
systems have been scarcely investigated. Now it is possible to discern in a general case only two very different states and two corresponding stages of the nonstationary evolution: a) strongly nonlinear nonstationary stage when we have violent relaxation [4], accompanied by "simulated mixing" $[1,2]$; in this case the chaos and chaotic motions of stars play a very important role, (b) weakly nonstationary state or quasilinear case, when "quasidiffusion mixing" [2] takes place; in this case any types of instability play an important role. We think there must be at least one more intermediate state between these two states, (c) with a moderate degree of nonstationarity. In this case it is hard to predict exactly the corresponding kind of phase mixing. However, it is evident that here the Lynden-Bell's statistics and his distribution function are absolutely invalid ( $t$ his distribution function and the statistics do not occur in some real strongly nonlinear nonstationary states, even in case (a) (see articles of A.M .Fridman etc. $(1983,1989))$. If relaxation due to the compulsive mixing takes place in the field of regular forces of the whole system, then at the moderate nonstationary state for the relaxation process small-scale interactions of various massive collective formations with stars and their streams are important as well. Therefore, the corresponding kind of mixing (in the case (c)) may be conveniently called, for example, as a semiforced one (or a moderate mixing). A detailed research of phase mixing mechanisms and the analysis of the role of instabilities requires numerical calculations and building of phase models of non-stationary systems.

\section{CHAOTIC PHENOMENA IN THE PHASE-SPACE}

The gravitation field in strongly nonstationary stellar system is abruptly changing not only in time, but also in space with a varying gradient to lead to the simulated mixing in phase space. Therefore, stars which are born in this process are feeling "push" and are moving chaotically. If we take some element of the phase volume, the simulated mixing gives rise to statistical stretch of this element by the exponential law. This process reminds of the classical problem of mapping the $\mathrm{N}$-dimensional sphere itself.

The mechanism of statistical stretch can be simulated as follows. Let the impact on the phase element at time $t$ be described by the matrix $M_{n}$, where $n=t / \tau, \tau$ being $t$ he mean time interval, within which two successive impacts can be considered to be random. Then the degree of the phase element stretch at $t$ ime $t$ is characterized by the eigenvalues of the matrix

$$
R_{n}=M_{n} M_{n-1} \cdots M_{1}
$$

where $M_{i}$ are random matrices. Indeed, if in the initial state there is a sphere 


$$
\overrightarrow{\mathrm{q}}^{2}=1, \quad \overrightarrow{\mathrm{q}}=\overrightarrow{\mathrm{q}}\left(\mathrm{x}, \mathrm{y}, \mathrm{z}, \mathrm{v}_{\mathrm{x}}, \mathrm{v}_{\mathrm{y}}, \mathrm{v}_{\mathrm{z}}\right)
$$

then after the first impact we have $\vec{q}_{1}=M_{1} \vec{q}$. Substituting $\vec{q}=M_{1}^{-1} \vec{q}_{1}$ into (2), we obtain

$$
\vec{q}_{1}\left(M_{1} M_{1}^{T}\right)^{-1} \quad \vec{q}_{1}=1,
$$

where the symbol $T$ means matrix transposition. This is the equation of ellipsoid, hence, its semi-axes are characterized by eigenvalues of the matrix $M_{1}$ and so on.

That is why we have done numerical simulation to observe an asymptotic behaviour of the product of the random matrix $R_{n}$, making use of the generator of random numbers, 1ying in the interval $(0,1)$. Let $M_{i}=A(k)$ with the probability $p$ and $M=B(d)$ with the probability 1-p, where $A(k)$ is the deformation matrix, $B(d)$ is the rotation matrix,

$$
\begin{aligned}
& \mathrm{AB} \neq \mathrm{BA}, \quad \overrightarrow{A \mathrm{q}} \neq \overrightarrow{\mathrm{Bq}} \neq \overrightarrow{\mathrm{q}} \\
& |\operatorname{det} \mathrm{A}|=|\operatorname{det} \mathrm{B}|=1
\end{aligned}
$$

Then the volume of the initial system is constant, despite of the strong deformation. Let us denote eigenvalues of the matrix $M_{n} v i a \lambda_{n j}(j=1,2, \ldots)$ and $\lambda_{n \cdot 1}>\lambda_{n 2}>\ldots>\lambda_{n \mu}$, where $\mu$ is dimension of the system. Then the values

$$
n_{n i}=\frac{1}{n} \ln \left(\lambda_{n i} / \lambda_{n i+1}\right)
$$

define the rate of the volume stretch in time. To determine statistical characteristics of the process we need to repeat the calculation of $n_{n i}$ a lot of times and to average over the number of realizations $v$. We calculated the value of $\eta_{n i}$ and its dispersion $\sigma_{i}(n)$ over $v$ for each $n$ for various values of $\mu=2,4,6$ ( $\mu=2$ is the model of a homogeneous plane layer of finite thickness that oscillates in the $z$-direction; $\mu=4$ is the model of a disk oscillating in the plane $(x y) ; \mu=6$ is a spherical model being the most complicated case for calculation).

According to our calculations the curve $\sigma_{\dot{t}}(n)$ shows, at the beginning, chaotic oscillations lacking relaxation. Here 
chaos takes comparatively little time. The stochastic character of the process al ways turns to the regime of relaxation. It is of interest to compare these results with the central limit theorm of the probability theory, according to which for a number of assumptions on the continuity of some functions $\sigma_{i}(n) \sim n^{-1 / 2}$ should be satisfied. Our setting of the problem lacks such assumptions, that is why our results differ essentially from the law $(-1 / 2)$. But all this, as well as the question on a specific application of the results to real objects is the topic of a separate examination.

Finally note that the method proposed here is in principle the extension to the multidimensional case of the transformation over the angle $\theta_{n}=\Phi\left(\theta_{n-1}\right)$, that was considered in Ref. [5] in the one-dimensional version for other problems. We do consider the vector transformation, but a stochastic character of the physical process is associated with collisionless relaxation. In fact, the interaction of largescale condensations or density waves themselves and with each other was well as other nonstationary processes take place.

\section{THE QUASIDIFFUSION MIXING}

In the case when nonstationarity of the stellar system is weak, relaxation generally proceeds due to quasidiffusion mixing [2]. Here any instability can turn the evolution to quite a new branch to activate strongly quasidiffusion. While for forced mixing the distance between two points in the phase space increases exponentially, then for quasidiffusion it increases by the power law.

Our calculations show that the periodic nature of the perturbed force frequently leads to a resonance phenomenon. Indeed, expanding the perturbation potential

$$
U(r, t)=U_{0}(r)+\sum_{k} \varepsilon^{k} \psi_{k}(r) \cos (k \omega t)
$$

and the velocity of movement

$$
v(r, t)=v_{o}(r)+\sum_{k} \varepsilon^{k} w_{k}(r, t)
$$

( $\varepsilon$ is the perturbation amplitude and $\varepsilon \ll 1$ ), from the evolution equation for $v(r, t)$ [6] one can $f i$ nd $t$ he corresponding unknown functions $w_{k}(r, t)$ for preset $U_{0}(r)$ and $\psi_{k}(r)$. Then when certain resonance relations similar to the direct Landau dampling are met, quasidiffusion $w_{k} \sim t^{k}$ occurs. Note, 
that if the rotation of the initial model is taken into account, the direct Landau damping during resonance can be replaced by the reverse damping in some cases.

Quasidiffusion is optionally associated with resonances, since we can consider the effect of more or less random perturbations of small amplitudes almost in a similar way. Moreover, quasidiffusion occurs due to the interaction of individual stars with different stellar or gas condensations. The role of such condensations may be played not only by material objects, but also by density waves. The nature of condensations is not important for us. The results of exact calculations for different condensations will be published elsewhere.

\section{NONSTATIONARY MODELS INSTABIL IT IES}

One of the most interesting problems is that of building exact nonequilibrium models of nonstationary stellar systems, finding their phase space density. This trend in the investigations is particularly necessary for the study of moderate nonstationary states and discovery of new kinds of instability occuring due to initial nonstationarity.

This leads us to the solution of the problem on the stability of a nonequilibrium state.

At present we have constructed, for example, the following nomlinearly pulsating models:

a) a pulsating version of the equilibrium model of the Einstein sphere $[7,8]$;

b) a pulsating version of the Camm's equilibrium model [9, $10]$

c) rotational versions of models (a) and (b) [11];

d) a rotational pulsating MacLauren disk with the isotropic velocity diagram [12];

e) a pulsating disk with the anisotropic velocity diagram [12];

All six models have been extensively studied in Refs. [7-13] from the point of view of their stability. We derived some new kinds of instabilities (see [7-13]).

\section{REFERENCES}

[1] Antonov, V.A., Nuritdinov, S.N., Osipkov, L.P. (1973), Dynamics of galaxies and stellar clusters, Nauka, AlmaAta, $p .55$.

[2] Nuritainov, S.N., (1979), Astron. Tsirkul. Acad. Sc. USSR, No. $1081, \mathrm{p} .1$.

[3] Nuritainov, S.N. (1989), Problems of Phys, and Dyn. Stell. Systems, Tashkent, p.46. 
[4] Lynden-Bell, D. (1967), Month. Not.R.A.S., V.136, p.101.

[5] Antonov, V.A.(1984), Vestnik of Leningrad University, No. 7, p.67.

[6] Nuritainov, S.N. (1975), Astrophysika, V.11,p.135.

[7] Antonov, V.A., Nuritdinov, S.N. (1981), Sov.Astron.J. $\mathrm{V} .58, \mathrm{p} .1158$.

[8] Nuritdinov, S.N. (1985), Sov. Astron.J., V.62,p.506.

[9] Nuritdinov, S.N. (1983), Sov. Astron.J., V.60,p.40.

[10] Nuritdinov, S.N. (1991), Sov. Astron.J. (to be pub1 ished).

[11] Nuritdinov, S.N. (in press).

[12] Nuritdinov, S.N. (1987), Dynamics of gravit.systems and methods of analytic Celestial Mechanics, Nauka, Alma-Ata, p.65.

[13] Nuritdinov, S.N. (1988), Astron. Tsirk. Acad.Sc.USSR, No. 1531, p.5. 